\title{
Stem cell therapy for a debilitating retinal disorder
}

Retinitis pigmentosa is a devastating condition of the eye's light-detecting photoreceptor cells. The disease leads to severe vision impairment and eventual blindness. One hopeful avenue of therapeutic research is the development of retinal sheet transplants that could be grafted into the eyes of human patients. However, progress has been impeded, partly owing to the lack of an appropriate animal model of the disease, as well as common difficulties associated with stem cell grafts. A new study by Shirai et al. (Proc. Natl. Acad. Sci. USA 113, E81-E90; 2015) presents two novel primate models of retinitis pigmentosa, and an evaluation of viable retinal transplants using tissue derived from human embryonic stem cells.

In the study, researchers created retinal sheet transplants from human stem cells that successfully developed cell structures containing rhodopsin, the light-sensitive protein of our eyes. The transplants were then placed in immunosuppressed rats, and after validating the technique, the researchers then generated two primate models of

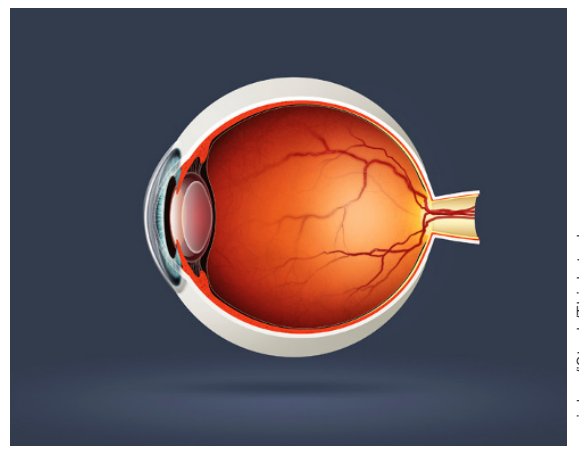

retinitis pigmentosa. In one model, Shirai and colleagues used a cobalt-chloride injection that successfully caused degeneration of the retinal layers containing photoreceptor cell bodies. In a second model, the researchers applied a laser to selectively injure this same retinal layer. Both models were examined with fine-scale imaging of the tissue injuries and electroretinograms to measure the electrical responses of photoreceptors. After confirming that these models do indeed mimic the characteristics of retinitis pigmentosa, the authors transplanted the stem cell-derived retinal grafts.
Importantly, the primate models were given immunosuppressants, and the implanted retinal grafts showed no signs of transplant rejection. Further, under high-resolution examination, the grafts were observed to increase in thickness over the course of the experiments. While the electroretinograms did not show recovery of photoreceptor activity, imaging demonstrated that cells from the host eye did create contacts with cells from the transplant. Thus, the stem cell-derived retinal graft both grew inside the host eye and appeared to integrate into the neural tissue of the animal, giving new hope to the possibility of using such grafts in human patients in the future.

Animal models and stem cell techniques are a promising pathway to curing not only retinitis pigmentosa, but many other forms of retinal degeneration. This demonstration that human embryonic stem cells can be used to partially repair damaged retinas is an exciting step in the search for a treatment therapy for all degenerative disorders. James E. Niemeyer

\section{MOUSE MODELS REVEAL WHY ALZHETMER'S TREATMENT FATLS}

Alzheimer's disease is an infamous and debilitating neurological disorder that causes profound memory loss. A major physical characteristic of the disease is the buildup of amyloid- $\beta$ plaques in the brains of afflicted patients. Scientists have constructed antibodies that can specifically target and attack these plaques in diseased brains, and application of these antibodies results in decreased presence of amyloid- $\beta$ plaques in humans; however, these treatments often still fail to confer cognitive benefits to patients.

A recent study by Busche et al. (Nat. Neurosci. 18, 1725-1727; 2015) examined this conundrum in a mouse model of Alzheimer's disease. The authors used transgenic mice that overexpress a mutated human amyloid precursor protein, producing higher concentrations of amyloid- $\beta$ in the neocortex. The researchers then applied the monoclonal antibody 3D6, which was developed to attack amyloid- $\beta$ plaques. 3D6 is the mouse equivalent of bapineuzumab, a humanized antibody that has been unsuccessfully employed to treat human Alzheimer's patients.

The authors confirmed that the immunotherapy did result in decreased presence of amyloid- $\beta$ plaques. However, using two-photon calcium imaging in these treated mice, the researchers discovered significant neurophysiological problems. They found that a large proportion of neurons had hyperactive calcium signals, and in nearly half of the treated mice, the researchers also found an unusually large amount of neuronal synchrony. Such synchrony is characteristic of seizures, and this could help promote the onset of epilepsy that is seen in many mouse models of Alzheimer's disease. Importantly, when the 3D6 antibody was administered to wild type mice, the animals did not display this hyperactivity in calcium signals. Therefore, these neurophysiological symptoms likely result from a combination of mice overexpressing the amyloid precursor protein and the application of the 3D6 antibody.

The discovery that amyloid- $\beta$ plaques can be reduced via passive immunotherapy with antibodies like bapineuzumab has led to much excitement about a potential cure for Alzheimer's disease. However, the discovery that these antibodies do not ameliorate the symptoms of patients has been disheartening. The study by Busche et al. provides important insight for treatments of Alzheimer's disease, and shows that the process of repairing brain dysfunction is complicated, often resulting in unintended consequences. Studies such as this are key to gaining a better understanding of Alzheimer's disease and will lead to more focused efforts to develop therapies that combat both the anatomical and neurophysiological symptoms of the disorder.

James E. Niemeyer 